\title{
DETERMINACIÓN DE FACTORES DE PROTECCIÓN PARA MASTITIS BOVINA EN FINCAS ADMINISTRADAS BAJO EL SISTEMA DOBLE PROPÓSITO EN EL MUNICIPIO DE MONTERÍA
}

\section{DETERMINATION OF PROTECTION FACTORS FOR BOVINE MASTITIS IN FARMRS MANAGED UNDER THE DOUBLE PURPOSE SYSTEM IN THE MUNICIPALITY OF MONTERIA}

\author{
Alfonso Calderón ${ }^{1}$, Nicolás Martínez², José Cardona ${ }^{3}$
}

\begin{abstract}
${ }^{1}$ MVZ, M.Sc. Docente. Instituto de Investigaciones Biológicas del Trópico (IIBT), Facultad de Medicina Veterinaria y Zootecnia, Universidad de Córdoba. Kilómetro 27 vía Ciénaga de Oro. Correspondencia: alcaran1@yahoo.com, ${ }^{2}$ MVZ, Esp. Docente. Facultad de Medicina Veterinaria y Zootecnia, Universidad de Córdoba. Kilómetro 27 vía Ciénaga de Oro. Correspondencia: nicom126@gmail.com, ${ }^{3 \cdot}$ MVZ, M.Sc. Docente. Facultad de Medicina Veterinaria y Zootecnia, Universidad de Córdoba. Kilómetro 27 vía Ciénaga de Oro. Correspondencia: cardonalvarez@hotmail.com
\end{abstract}

Rev. U.D.C.A Act. \& Div. Cient. 12 (2): 61-68, 2009

\section{RESUMEN}

Para determinar factores de protección para mastitis, se aplicaron encuestas, con el fin de evaluar las diferentes prácticas implementadas en la prevención, el control y el manejo de la mastitis bovina, en 15 fincas manejadas bajo el sistema doble propósito, en el municipio de Montería (Colombia). El diagnóstico de la mastitis bovina, se realizó por medio del CMT, RCS y AB. Las diferentes variables se cruzaron con el resultado de las pruebas diagnósticas utilizando tablas de contingencia y para determinar la asociación, se implementó la prueba de Chi-cuadrado $\left(\mathrm{X}^{2}\right)$ y se calculó la razón de disparidad u Odds Ratio (OR), con su respectivo intervalo de confianza (95\%). Como factores de protección generales, se encontraron la administración directa de los propietarios y la permanencia de éstos cuando fue superior al $50 \%$ de su tiempo. En la rutina del ordeño, la división del trabajo por los operarios, el apoyo del ternero, la implementación de la higienización de los pezones, el secado aceptable de éstos y el sellado o el amamantamiento de los terneros al final del ordeño, fue positivo. El descarte de vacas con mastitis durante el último año, la aplicación de la terapia de la vaca seca, la desinfección de la punta del pezón antes de esta terapia y la entrega de recomendaciones sanitarias por escrito para mastitis, dentro de las prácticas de prevención, se identificaron como factores de protección dentro de los aspectos sanitarios.

Palabras clave: Factores de protección, mastitis, doble propósito.

\section{SUMMARY}

To determine the protection factors for mastitis, questionnaires were applied to determine the control measures carry out for prevention and management of the bovine mastitis in 15 farms, managed under a double purpose system in the municipality of Monteria (Colombia). The diagnosis of the bovine mastitis was established by the CMT, RCS and bacteriological cultures (isolation). Variables were crossed with the results of the different diagnosis test using contingency charts. To determine the association, a Chi-square test and the Odds Ratio (OR) with their respective confidence interval 
(95\%) were calculated. As general protection factors the direct administration by the owners and a permanency higher than $50 \%$ of the time was found. During milking, the division of work by the milkers regarding the milking procedure, the support of the calf, the implementation of sanity of nipples, when drying of nipples was acceptable, nipple sealing and the suckling of the calves at the end of milking were positive. The culling of cows with mastitis during the last year, the application of the dry cow therapy, the disinfection of the tip of nipples previous the dry cow therapy and the written delivery of sanitary recommendations were identified as protection factors in the aspects of practices of prevention.

Key words: Protection factors, mastitis, double purpose.

\section{INTRODUCCIÓN}

La producción de leche en Colombia para el 2007 fue de 6.167 millones de litros/año, de los cuales, la región Caribe, junto con los departamentos Santander y Norte de Santander, aportaron el 24,81\% de la producción nacional. Córdoba, con Antioquia y con Meta concentraron los mayores inventarios ganaderos en Colombia. A nivel regional, el departamento de Córdoba contribuyó con el 14,62\% y aportó el 3,63\% a la producción nacional (Minagricultura, 2007; Viloria, 2004).

En Córdoba, se presenta un desconocimiento de la calidad fisico-química y microbiológica de la leche fresca y de los productos lácteos producidos en las diferentes zonas del departamento destinada para consumo humano; los últimos estudios publicados (Calderón et al. 2006; Calderón et al. 2007) arrojan valores superiores o inferiores respecto a los mínimos establecidos en el decreto 616 de 2006, expedido por el Ministerio de la Protección Social (Minprotección, 2006).

Los factores de protección, se consideran aquellos elementos, atributos, características individuales o ambientales asociados con la disminución o la atenuación en la probabilidad que aparezca una enfermedad, aunque no implican, necesariamente, una relación causa-efecto; a su vez, sirven como moderadores de los factores de riesgo y la cuantificación del grado de riesgo constituye un elemento esencial en la formulación de planes de prevención y de control, ya que estos métodos excluyen a la intuición y a la casualidad (Clayton,1992). La mastitis, se manifiesta cuando los diferentes factores, como agente, hospedero y ambiente interactúan, ya sea para aumentar la exposición de la ubre a los microorganismos o para reducir la resistencia del hospedero o ayudar a los patógenos a colonizar el canal del pezón (CNM, 1990).

El sector agropecuario es la principal actividad económica de este departamento y soporte económico de los diferentes municipios. En Córdoba no existen reportes sobre las buenas prácticas del ordeño, lo que dificulta garantizar la calidad de la leche producida. El objetivo del presente trabajo fue determinar y cuantificar las prácticas de manejo, de prevención y de control para la mastitis bovina, que se constituyeron en factores de protección para la disminución del riesgo de mastitis bovina en fincas administradas bajo el sistema doble propósito, en el municipio de Montería (Córdoba).

\section{MATERIALES Y MÉTODOS}

Sitio de estudio: Este trabajo se adelantó en el área rural de la ciudad de Montería, capital del departamento de Córdoba, al noroeste de Colombia, situada a $8^{\circ} 45^{\prime}$ de latitud norte y $75^{\circ} 53^{\prime}$ de longitud oeste, con una altitud de $18 \mathrm{msnm}$ y ubicada en el valle medio del río Sinú, con una extensión de $3.043 \mathrm{~km}^{2}$, el clima es cálido tropical con una estación de sequía y una de lluvias a lo largo del año y una precipitación anual de 1200 a $1500 \mathrm{~mm}$. La temperatura promedio anual es de $28^{\circ} \mathrm{C}$, con picos mínimos-máximo de $16-35^{\circ} \mathrm{C}$, en época lluviosa y de $22^{\circ} \mathrm{C}$ hasta $45^{\circ} \mathrm{C}$, en temporada de sequía; la humedad relativa es del $85 \%$ y está ubicado dentro de la franja ecológica bosque seco tropical (bst) y en la zona agroecológica Cj (Corpoica, 2002); de topografía básicamente plana, con pendientes hasta del 3\%, tierras de planicies aluviales sujetas a inundaciones periódicas durante el año; con suelos formados a partir de materiales sedimentarios que registran poca evolución y mal drenaje (IGAC, 1992). Los pastos predominantes son admirable (Brachiaria mutica), braquipará (Brachiaria sp.) y angleton (Dichantium aristatum); además, existen leguminosas nativas (Corpoica, 2002). Cuenta con una población ganadera de 489.036 bovinos (Fedegan, 2007), donde predominan los bovinos de doble propósito con los cruces Bos taurus (Holstein, Pardo Suizo, Simmental y criollo) y Bos indicus (Brahmán, Gyr), en diferentes proporciones (Corpoica, 2002).

Tipo de estudio y tamaño de la muestra: Se realizó un estudio epidemiológico transversal, de tipo descriptivo, 
en fincas manejadas bajo el sistema doble propósito, escogidas mediante un diseño por conveniencia, donde se buscó que éstas presentaran condiciones de manejo similares, facilidad para el muestreo, acceso a registros de salud, de producción y el deseo de participar en el estudio por parte de los ganaderos.

Para averiguar la situación de la mastitis bovina y sus factores de protección, se llevó a cabo visitas 15 fincas, donde se evaluaron 1.065 vacas, para conocer el estado de salud de la glándula mamaria, por medio de la prueba California para mastitis (CMT), recuento de células somáticas (RCS) y examen bacteriológico (EB).

Para la prueba del CMT, se siguió la metodología propuesta por Sargeant et al. (2001) y se tomaron como cuartos positivos las reacciones a partir de trazas, hasta grado; de los pezones positivos al CMT, se recolectó una muestra de $5 \mathrm{~mL}$ de leche en un tubo de vidrio estéril con tapa rosca, identificando la muestra con el número o nombre de la vaca y del pezón en el tubo. Posteriormente, se lavó con una solución de yodo el pezón, luego fue secado con papel desechable y se desinfectó el esfínter del pezón con torundas de algodón impregnadas con alcohol antiséptico, hasta que la última torunda, una vez usada, saliera blanca; igual procedimiento se siguió para la toma de las muestras de los casos de mastitis clínica, con el propósito de garantizar la asepsia de las muestras, las cuales, se conservaron en refrigeración hasta el procesamiento en el laboratorio del Instituto de Investigaciones Biológicas del Trópico (IIBT), de la Universidad de Córdoba.

Una alícuota de la muestra, se sembró en agar sangre y agar Mc Conkey y la identificación por género y por especie, se siguió la metodología propuesta por el consejo nacional de la mastitis de Estados Unidos (NMC, 2005). Para el RCS, se tomó una alícuota de $60 \mu \mathrm{L}$ por medio de un dispositivo comercial, el cual, contiene unos capilares impregnados con una sonda fluorescente marcada con yoduro de propidio que se une específicamente al ADN de las células; luego, el dispositivo es colocado en la cámara de lectura del equipo, que contabiliza la producción de señales fluorescentes (DeLaval, 2003).

En esa misma visita, se aplicó una encuesta con 101 preguntas, algunas se evaluaron por observación directa durante el ordeño y otras por entrevistas al personal de apoyo, administradores y propietarios, con el objetivo de determinar qué prácticas estaban implementando en la prevención y control de la mastitis bovina.

La información obtenida correspondiente a las variables de la finca y a las variables de las vacas y se cruzó en paralelo con el resultado de las pruebas diagnósticas (CMT, RCS y EB). Se consideró una vaca positiva al diagnóstico de mastitis cuando, al menos en uno de sus cuartos, fue aislado algún germen etiológico, o cuando el RCS fue > a $297.500 \mathrm{CS} / \mathrm{mL}$ o cuando a la prueba del CMT, al menos en un cuarto, fue positivo. La prueba de Chicuadrado $\left(\mathrm{X}^{2}\right)$, se aplicó para determinar la probabilidad de que las diferencias observadas en la población no sean debidas únicamente al azar, sino para que exista una verdadera asociación (diferencia real) entre el resultado del diagnóstico y la característica evaluada. Por convención, cuando la probabilidad fue menor del $5 \%$ (p-valué $<0,05$ ), se dice que las tasas son significativamente diferentes, por lo tanto, el factor y la enfermedad están estadísticamente asociados (Martín et al. 1987).

Se determinó la razón de disparidad u Odds Ratio (OR) con su respectivo intervalo de confianza del $95 \%$, que demuestra el grado o fuerza de asociación entre los factores de protección y la presentación de mastitis. Se consideró que valores de OR inferiores a uno son factores de protección frente a la presentación de una enfermedad (Martín et al.1987). Para estos cálculos, se empleó el programa estadístico para las ciencias sociales (SPSS).

\section{RESULTADOS Y DISCUSIÓN}

Los factores que interactuaron y que se convirtieron en factores de protección para la presentación de mastitis, se presentan en la tabla 1.

Al evaluar el tiempo de permanencia que los propietarios dedicaron a la administración de las fincas, se halló que en el $74 \%$ ( $n=11)$ de los casos, los propietarios dedicaron menos del $50 \%$ de su tiempo a la administración, en comparación con el $26 \%(n=4)$, que permanecieron más del $50 \%$ de su tiempo; esta mayor dedicación de su tiempo hizo que las vacas presentaran 0,64 veces menos de probabilidad de presentar mastitis, debido, posiblemente, a los mayores cuidados y esfuerzos de los ganaderos en disminuir los efectos adversos de las enfermedades y a disminuir los costos de producción. 
Tabla 1. Factores de protección para mastitis bovina en explotaciones manejadas bajo el sistema doble propósito en el municipio de Montería.

\begin{tabular}{|c|c|c|c|c|c|}
\hline \multirow{2}{*}{ Variable } & \multirow{2}{*}{$X^{2}$} & \multirow{2}{*}{ Valor de $p$} & \multirow{2}{*}{ OR } & \multicolumn{2}{|c|}{ Limite } \\
\hline & & & & Inf. & Sup. \\
\hline Permanece más del $50 \%$ en la finca & 6,45 & 0,011 & 0,64 & 0,45 & 0,90 \\
\hline Los ordeñadores no son los mismos que manean & 19,65 & 0,000 & 0,55 & 0,42 & 0,71 \\
\hline El ordeño se hace con apoyo del ternero & 21,05 & 0,000 & 0,40 & 0,27 & 0,60 \\
\hline Se hace higienización de los pezones & 2,33 & 0,000 & 0,81 & 0,63 & 0,90 \\
\hline El agua del lavado se le adiciona un desinfectante & 31,42 & 0,000 & 0,30 & 0,19 & 0,46 \\
\hline Secan lo lavado o presellado & 21,60 & 0,000 & 0,30 & 0,18 & 0,51 \\
\hline La prueba del guante blanco es aceptable & 8,28 & 0,000 & 0,32 & 0,12 & 0,48 \\
\hline Sellan o traer el ternero al final del ordeño & 21,05 & 0,000 & 0,40 & 0,27 & 0,60 \\
\hline Descartaron vacas por mastitis durante el último año & 3,12 & 0,000 & 0,76 & 0,56 & 0,98 \\
\hline El Médico Veterinario deja recomendaciones por escrito & 7,76 & 0,050 & 0,44 & 0,11 & 0,88 \\
\hline Realizan el CMT & 1,06 & 0,030 & 0,77 & 0,67 & 0,93 \\
\hline Aplican la terapia de la vaca seca (TVS) & 23,03 & 0,000 & 0,50 & 0,38 & 0,67 \\
\hline Desinfección la punta del pezón pre-TVS & 4,95 & 0,026 & 0,58 & 0,36 & 0,94 \\
\hline Se amputan pezones supernumerarios & 12,31 & 0,000 & 0,54 & 0,38 & 0,76 \\
\hline
\end{tabular}

La implementación de prácticas de higienización de los pezones, como el lavado o el presellado y el secado de estos, reducen y/o eliminan la concentración de los microorganismos en la piel; en caso de no efectuasen estos procedimientos se incrementa el riesgo de infectarsen las glándulas y padecer de mastitis (Philpot $\varepsilon$ Nickerson, 2000) o de contribuir con altos recuentos de mesófilos, lo cual disminuye la calidad microbiológica de la leche (Calderón et al. 2006).

En fincas, donde se implementó el lavado de los pezones y si al agua se le adicionó un desinfectante, como yodo, principalmente, las vacas mostraron 0,30 veces menos posibilidades de infectarse, ya que este desinfectante, en muchas ocasiones, mejora la calidad microbiológica del agua de lavado. Burmeister et al. (1998) hallaron una mayor efectividad antibacterial del presellado a diferencia del simple lavado, por el efecto germicida del producto empleado.

El ordeño con la división del trabajo y las personas que ordeñaron no manearon o sujetaron las patas de las vacas, arrojó un resultado de 0,55 veces menos probabilidades de infectar las glándulas mamarias, posiblemente, a raíz de la mayor limpieza de las manos de los ordeñadores. En el sistema doble propósito, por la presencia de genes
$B$. indicus, es necesario garantizar la seguridad de estos operarios. También en este sistema es necesario el apoyo del ternero para producir la eyección de la leche y que una vez finalizado el ordeño, beban, de un pezón más, la leche residual y las vacas permanezcan de pie por más tiempo; esta práctica de ordeño con ternero arrojó como resultado 0,40 veces menos de probabilidad de presentar mastitis, en comparación con fincas en donde no se ordeñó con apoyo y no se hizo el sellado de los pezones, lo que permite que las glándulas mamarias se infecten por el no cierre del esfínter y de la colocación de una barrera antibacteriana, cuando se hace el sellado con yodo (Blowey \& Edmonson, 1999).

Con la práctica de la higienización de los pezones se hace necesario efectuar el secado de los mismos; ejercicio que se realizó en el $47 \%(n=7)$ de las fincas, lo que hizo que las vacas presentaran 0,30 veces menos posibilidades de originar mastitis. Calderón et al. (2008) encontró que en el $90 \%(n=18)$ de las fincas de los valles de Ubaté y de Chiquinquirá se llevan a cabo prácticas de higienización, que incluyen el lavado o presellado y el secado de lo higienizado. Philpot $\&$ Nickerson (2000) afirmaron que cuando se implemente una rutina de ordeño que involucre lavado o presellado de los pezones, 
se debe establecer el secado de éstos, dentro del plan integral de control de la mastitis bovina.

El sellado de los pezones, al final del ordeño o colocar al ternero para que mame los pezones y tome la leche residual, está contribuyendo para que los patógenos infecciosos y ambientales no puedan colonizar el interior de la ubre, debido a que los selladores forman una barrera entre la piel del pezón y el ambiente (Philpot $\mathcal{E}$ Nickerson, 2000; Chacón et al. 2006) o haciendo que las vacas permanezcan más tiempo de pie debido al mamado de la leche residual por los terneros evitando que estas se acuesten aún con sus esfínteres abiertos sobre superficies altamente contaminadas.

Novoa et al. (2005), en Cuba, determinaron que una inadecuada rutina de ordeño representa una gran importancia en la aparición de mastitis bovina y Calderón et al. (2008), en los valles de Ubaté y de Chiquinquirá, hallaron que solamente el $75 \%(n=15)$ de los ganaderos y asistentes técnicos evaluados organizaron, correctamente, los pasos para realizar una excelente rutina de ordeño y que éstos le dan más significación a la velocidad que a la calidad con que se hace este trabajo, coincidiendo con lo afirmado por Ferguson et al. (1994), donde concluyeron que las infecciones mamarias en los hatos lecheros se deben a rutinas no adecuadas.

El descarte de vacas a causa de mastitis durante el último año hizo que estas presentaran 0,76 veces menos posibilidades de suscitar mastitis; igualmente Cassel et al. (1994) afirmaron que el descarte de vacas con base en los resultados de cultivo es un mecanismo de control para S. aureus. Saran E Chaffer (2000) recomiendan eliminar vacas de más de tres lactancias infectadas crónicamente con $S$. aureus, que no responden al tratamiento con antibacterianos, así como vacas con $\mathrm{RCS}>500.000 \mathrm{cel} / \mathrm{mL}$, durante períodos prolongados, ya que son hospederos crónicos de patógenos contagiosos y aumentan los niveles de las células somáticas en la leche del tanque. Así mismo, Philpot E Nickerson (2000) recomiendan la eliminación de vacas con infecciones crónicas dentro del plan integral de control de mastitis.

Cuando el asistente técnico entregó las recomendaciones para la prevención y el control de la mastitis, en forma escrita, se presentaron 0,44 veces menos problemas de infección mamaria. A pesar que el grado de escolaridad de los mayordomos de las fincas fue bajo, ya que el $67 \%(n=10)$ cursó la primaria y el 33\% $(n=5)$ algunos grados de bachillerato, es posible que los mayordomos o administradores estén supervisando los tratamientos recomendados por los asistentes técnicos o verifiquen que éstos se implementen en las diferentes labores, siguiendo el protocolo propuesto por el asesor.

En sistemas especializados de leche, como son los valles de Ubaté y de Chiquinquirá, solamente el $45 \%(n=9)$ de los ganaderos y asistentes técnicos implementaron la prueba del CMT, como un diagnóstico rutinario y el $55 \%$ $(n=11)$ de los casos que no la realizaron, se debió que no estaban siendo motivados, económicamente, a producir leche de mejor calidad (Calderón et al. 2008). Cuando este método se instauró en las fincas evaluadas del sistema de doble propósito, se determinó que solamente el $40 \%(n=6)$ efectuó esta prueba, y esto hizo que las vacas presentaran 0,77 veces menos posibilidades de infectar sus glándulas mamarias; los mayordomos evaluados consideraron esta práctica muy importante en la prevención y el control de la mastitis. Kirk et al. (1994) observaron que la segregación de vacas con mastitis clínica y el ordeño al final resultó en una disminución en 0,81 veces menos las probabilidades de manifestar mastitis en vacas de lecherías especializadas.

La implementación de la terapia de la vaca seca (TVS), se efectuó en el $33 \%(n=5)$ de las fincas y esto ocasionó que las vacas presentaran 0,50 veces menos probabilidades de generar problemas de mastitis, Calderón et al. (2008), en los valles de Ubaté y de Chiquinquirá estableció que el $30 \%(n=6)$ de los ganaderos realizaron la TVS y consideran que esta es la mejor alternativa para finalizar una lactancia. Pankey et al. (1982), Erskine et al. (1987) y Erskine et al. (1992) demostraron que la implementación de la TVS a todas las vacas, al finalizar la lactancia, se asoció con un bajo RCS de tanque.

Es importante el manejo de medidas profilácticas antes de la inserción o aplicación de la cánula en la TVS; cuando se llevó a cabo la desinfección de la punta del pezón con productos germicidas, reveló un 0,58 veces menos probabilidades de padecer de mastitis. Los pezones se deben limpiar y desinfectar cuidadosamente antes de cualquier infusión, ya que los microorganismos en la punta del pezón pueden ser llevadas al interior de la ubre y producir infecciones principalmente por 
bacterias Gram negativos, ya que la mayoría de los productos han sido elaborados para eliminar agentes Gram positivos como S. aureus y contra estreptococos ambientales (S. uberis) pero carecen de actividad contra Gram negativos especialmente la E. coli, además, hoy día la industria farmacéutica recomienda la inserción parcial de la cánula, previa a la desinfección del esfínter del pezón, para garantizar la efectividad de esta práctica (NMC,2006).

Con estos resultados hallados, se puede concluir que la determinación y la cuantificación de los factores de protección o de riesgo para la mastitis permite clasificar las diferentes actividades de prevención y de control en buenas o malas prácticas ganaderas en el ordeño, siendo los primeros, bajo un conjunto de acciones, los que buscan garantizar al consumidor final de la leche y sus derivados, alimentos de excelente calidad, asegurando la inocuidad desde el eslabón primario, mediante la optimización del manejo, con el fin de volver más competitivo al productor ganadero.

Cuando la permanencia de los propietarios fue mayor al $50 \%$ de su tiempo y si el Médico Veterinario entregó recomendaciones escritas para la prevención y el control de la mastitis bovina, se consideraron como factores de protección dentro de los aspectos administrativos evaluados, lo que permitió asegurar que las estrategias para una producción más eficiente se están implementando, de acuerdo a parámetros preestablecidos y certificar los procesos.

La implementación de prácticas sencillas, como la sujeción de los miembros posteriores de las vacas por operarios diferentes a los ordeñadores, cuando el ordeño se realizó con apoyo del ternero, la implementación de la higiene de los pezones, el uso de una solución desinfectante para éstos, el secado aceptable de los pezones, el sellado de los pezones o el amamantamiento de los terneros al final del ordeño, el descarte de vacas por mastitis durante el último año, la implementación de la TVS y la desinfección de la punta del pezón antes de la TVS, se consideraron como factores de protección para la mastitis bovina en sistemas doble propósito, lo que permitió ofrecer una leche con exigencias del mercado, que mejoran la visión productiva de la empresa pecuaria.
AGRADECIMIENTOS: Los autores agradecen al director, a los investigadores y al personal de apoyo del Instituto de Investigaciones Biológicas del Trópico (IIBT) por la colaboración logística durante el desarrollo del proyecto. Conflictos de intereses: Este manuscrito fue preparado y revisado con la participación de todos los autores, quienes declaramos que no existe ningún conflicto de intereses que pongan en riesgo la validez de los resultados presentados. Financiación: Este estudio fue financiado por el Centro de Investigaciones de la Universidad de Córdoba.

\section{BIBLIOGRAFÍA}

1. BLOWEY, R.; EDMONSON, P. 1999. El control de la mastitis bovina en granjas de ganado de leche. Guía práctica e ilustrada. Editorial Acribia, Zaragoza (España). 208p.

2. BURMEISTER, J.E.; FOX, L.K.; HILLERS, J.K.; HANCOCK, D.D. 1998. Effects of premilking and posmilking teat desinfectans on teat skin condition. J. Dairy Sci. (USA). 81(7):1910-1916.

3. CALDERÓN, R.A.; GARCÍA, F.; MARTÍNEZ, G. 2006. Indicadores de calidad de leches crudas en diferentes regiones de Colombia. Rev. MVZ Córdoba. 11(1):725-773.

4. CALDERÓN, R.A.; RODRÍGUEZ. V.; VÉLEZ, S. 2007. Evaluación de la calidad de leches en cuatro procesadoras de quesos en el municipio de Montería, Colombia. Rev. MVZ Córdoba. 12(1):912-920.

5. CALDERÓN, R.A.; JIMÉNEZ, P.G.; GARCÍA, C.F. 2008. Determinación de buenas prácticas de ordeño en un grupo de gestión empresarial de ganaderos del altiplano cundiboyacense. Rev. U.D.C.A Act. \& Div. Cient. (Colombia). 11(1):143-152.

6. CASSEL, E.L.; VOUGH, L.R.; VALNER, M.A.; EICKELBERGER, R.C.; MANSPEAKER, T.E.; STEWART, L.E.; DOUGLAS, L.W.; PETER, R.R. 1994. A demostration project of interdisciplinary dairy herd extension. Advising finded by industry and user. 3 Impact on management. Practices. J. Dairy Sci. 77:2461-2476. 
7. CLAYTON, R.R. 1992. Transitions in drug use: Risk and protective factors. In: Glantz, M.; Pickeens, R. eds. Vulnerability to drug abuse. American Psychological Association, Washigton, DC (USA) p.15-51.

8. CONSEJO NACIONAL DE MASTITIS (CNM). 1990. Conceptos actuales de mastitis bovina. $3^{\text {a Edición. }}$ (USA). 47p.

\section{CORPORACIÓN COLOMBIANA DE INVESTIGACION} AGROPECUARIA (CORPOICA). 2002. Atlas de los sistemas de producción bovina. Modulo región Caribe. Plan de modernización tecnológica de la ganadería bovina colombiana. Bogotá, Colombia, 82p.

10. CHACÓN, A.; VARGAS, C.; JIMÉNEZ, M. 2006. Incidencia en el conteo de células somáticas de un sellador de barrera (yodo-povidona 0,26\%) y un sellador convencional (yoduro 0,44\%). Agronomía Mesoamericana (Costa Rica) 2:207-212.

11. DELAVAL. 2003. DeLaval cell counter DCC. De Laval International $\mathrm{AB}$. Tumba, Sweden. 32p.

12. ERSKINE, R.J.; EBERHART, T.R.J.; HUTCHINSON, L.J.; SPENCER, S.B. 1987. Herd management and prevalence of mastitis in dairy herds with high and low somatic cell counts. J. Am. Vet (USA). 190(11):1411-1416.

13. ERSKINE, R.J.; WILSON, R.C.; RIDDELL, R.G.; TYLER, J.W.; ASPEARS, H.J.; DAVIS, B.S. 1992. Intramammary admnistration of gentamicin as treatment for experimentally induced Escherichia coli mastitis in cows. Am. J. Vet. Res (USA). 53(3):375-381.

14. FERGUNSON, J.D.; GALLIGAN, D.T.; THOMSON, N. 1994. Principal descriptors of body condition score in Holstein cows. J. Dairy Sci. 77:26952703.

15. FEDERACION DE GANADEROS (FEDEGAN) - FONDO NACIONAL DEL GANADO (FNG). 2007. Índices de evaluación. Programa nacional de erradicación de la Fiebre Aftosa y Brucelosis
Bovina. Unidad regional de desarrollo ganadero de Córdoba. II Ciclo de vacunación. 40p.

16. INSTITUTO GEOGRÁFICO AGUSTÍN CODAZZI (IGAC). 1992. Córdoba características geográficas. Subunidad geográfica, Santafé de Bogotá, (Colombia). 121p.

17. KIRK, J.H.; DEGRAVES, F.; TYLER, J. 1994. Recent progress in treatment and control of mastitis in cattle. J. Am. Vet, Med. Assoc. (USA) 204:11521158.

18. MARTIN, W.S.; MEEK, A.H.; WILLEBERG, P. 1987. Veterinary epidemiology. Principles and methods. Iowa State U. press/Ames $1^{\text {a }}$ Ed. (USA). p.130139.

19. NATIONAL MASTITIS COUNCIL (NMC). 2005. Laboratory Handbook on bovine mastitis. Second printing infection. Verona. (USA). 222p.

20. NATIONAL MASTITIS COUNCIL (NMC). NMC factsheet-dry cow therapy 2006. Disponible desde Internet en: http://www.nmconline.org/drycow.htm (con acceso 09/07/09).

21. NOVOA, R.; ARMENTEORS, M.; ABELEDO, M.A.; CASANOVAS, E.; VALERA, R.; CABALLERO, C.; PULIDO, J. 2005. Factores de riesgo asociados a la prevalencia de mastitis clínica y subclínica. Salud Anim (Cuba). 27 (2):84-88.

22. MINISTERIO DE AGRICULTURA Y DESARROLLO RURAL (MINAGRICULTURA). 2007. unidad de seguimiento de precios de leche informe 002. Variables sistema de pago de la leche cruda al productor.

23. MINISTERIO DE PROTECCIÓN SOCIAL (MINPROTECCCIÓN). 2006. Decreto No. 616. Reglamento técnico sobre los requisitos que debe cumplir la leche para consumo humano que se obtenga, procese, envase, transporte, comercializa, expenda, importe o exporte en el país. 32p. Disponible desde Internet en: http://www.redlactea.org/decretos. htm (con acceso 05/05/09). 
24. PANKEY, J.W.; BARKER, R.M.; TWOMEY, A.; DUIRS, G. 1982. A note on effectiveness of dry cow therapy in New Zealand dairy herds. New Zealand Vet. J. 30(4):50-52.

25. PHILPOT, N.; NICKERSON, S. 2000. Ganando la lucha contra las mastitis. Naperville (USA). 192p.

26. SARAN, A.; CHAFFER, M. 2000. Mastitis y calidad de leche. Editorial Inter-Médica. Buenos Aires (Argentina). 196p.
27. SARGEANT, J.M.; LESLIE, K.E.; SHIRLEY, J.E 2001. Sensitivity and specificity of somatic cell count and California mastitis test for identifying intramamary infection in early lactation. J. Dairy Sci. 84:2018-2024.

Recibido: Octubre 21 de 2008

Aceptado: Agosto 13 de 2009 